\title{
Polycomb protein EZH2 regulates cancer cell fate decision in response to DNA damage
}

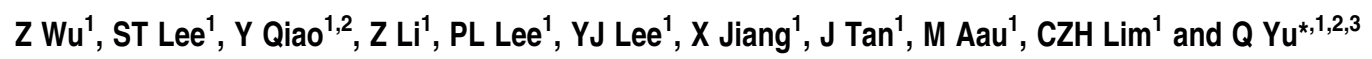

Polycomb protein histone methyltransferase enhancer of Zeste homologe 2 (EZH2) is frequently overexpressed in human malignancy and is implicated in cancer cell proliferation and invasion. However, it is largely unknown whether EZH2 has a role in modulating DNA damage response. Here, we show that EZH2 is an important determinant of cell fate decision in response to genotoxic stress. EZH2 depletion results in abrogation of both cell cycle G1 and G2/M checkpoints, directing DNA damage response toward predominant apoptosis in both p53-proficient and p53-deficient cancer cells, but not in normal cells. Mechanistically, EZH2 regulates DNA damage response in p53 wild-type cells mainly through transcriptional repression of FBXO32, which binds to and directs p21 for proteasome-mediated degradation, whereas it affects p53-deficient cells through regulating Chk1 activation by a distinct mechanism. Furthermore, pharmacological depletion of EZH2 phenocopies the effects of EZH2 knockdown on cell cycle checkpoints and apoptosis. These data unravel a crucial role of EZH2 in determining the cancer cell outcome following DNA damage and suggest that therapeutic targeting oncogenic EZH2 might serve as a strategy for improving conventional chemotherapy in a given malignancy.

Cell Death and Differentiation (2011) 18, 1771-1779; doi:10.1038/cdd.2011.48; published online 6 May 2011

Following DNA damage, mammalian cells activate cell cycle checkpoint mechanisms to induce cell cycle arrest and protect cells from apoptosis. ${ }^{1}$ The interconnections between the pathways regulating the cell cycle checkpoints and apoptosis dictate the cellular outcome to DNA damage, ${ }^{2}$ but whether these pathways are differentially regulated by oncogenic lesions in tumor cells to allow cancer-specific perturbation is poorly understood. Inhibition of key cell cycle checkpoint regulators such as cyclin-dependent kinase inhibitor p21 and Chk1/2 have been shown to increase the sensitivity to DNA damage in p53-proficient or p53-deficient cancer cells, respectively. ${ }^{3-14} \mathrm{~A}$ treatment strategy to simultaneously abrogate both $\mathrm{G} 1$ and G2/M checkpoint and thus sensitizing both p53 wild-type and mutant tumors has yet to be developed.

Polycomb protein enhancer of Zeste homologe 2 (EZH2) is a histone methyltransferase that is frequently overexpressed in a wide variety of human malignancies, ${ }^{15,16}$ and is implicated in cell proliferation, invasion and metastasis. ${ }^{17-25}$ Mechanistically, the oncogenic function of $\mathrm{EZH} 2$ has been attributed to associated histone $\mathrm{H} 3$ with trimethylated lysine 27 (H3K27Me3), leading to transcriptional repression of tumor suppressor genes, including p16(INK4a) and p19(ARF), ${ }^{26}$ E-cadherin, ${ }^{19}$ adrenergic receptor- $\beta 2,{ }^{27}$ RUNX3 ${ }^{20}$ p57 (also called CDKN1C), ${ }^{21} \mathrm{Bim}^{28}$ and DAB2IP. ${ }^{23,24}$ As such, EZH2 is emerging as a crucial regulator of cell fate by affecting multiple signaling pathways. It is not clear, however, whether EZH2 overexpression in cancer cells has a role in affecting cellular response to DNA damage. This study investigated the role of
EZH2 and associated epigenetic mechanism in regulating DNA damage checkpoints and apoptosis.

EZH2 depletion in cancer cells directs DNA damage response toward apoptosis by abrogating cell cycle checkpoints. To assess a potential role of EZH2 in DNA damage response, we depleted $\mathrm{EZH} 2$ by RNA interference in p53 wild-type osteosarcoma U2OS and colon cancer HCT116 cells, and treated the cells with DNA-damaging agents adriamycin (ADR) or etoposide (ETO). Although the control cells under above drug treatments exhibited mainly cell cycle arrest at both $\mathrm{G} 1$ and $\mathrm{G} 2 / \mathrm{M}$ phase, EZH2 knockdown cells exhibited a marked decrease in $\mathrm{G} 1$ arrest at $24 \mathrm{~h}$, followed by a loss of G2/M arrest and a robust increase of apoptosis at $48 \mathrm{~h}$ (Figure 1a and Supplementary Figure S1a). In addition, EZH2 knockdown also converted ADR-induced cell cycle arrest to apoptosis in p53-deficient cancer cells, including osteosarcoma Saos-2, colon cancer HCT116 p53 ${ }^{-/-}$and lung cancer $\mathrm{H} 1299$ cells (Figure $1 \mathrm{~b}$ and Supplementary Figures $\mathrm{S} 2 \mathrm{a}$ and $\mathrm{S} 2 \mathrm{c}$ ). Thus, EZH2 knockdown abrogates DNA damage-induced cell cycle arrest and promotes apoptosis in both p53-proficient and p53-deficient cancer cells.

EZH2 depletion abolishes both DNA damage-induced p21 and Chk1 activation. We next investigated the effects of $\mathrm{EZH} 2$ depletion on DNA damage checkpoint pathways in

\footnotetext{
${ }^{1}$ Cancer Biology and Pharmacology, Genome Institute of Singapore, Agency for Science, Technology and Research, Biopolis, Singapore; ${ }^{2}$ Department of Physiology, Yong Loo Lin School of Medicine, National University of Singapore, Singapore and ${ }^{3}$ Cancer and Stem Cell Biology, DUKE-NUS Graduate Medical School of Singapore, Singapore ${ }^{*}$ Corresponding author: Q Yu, Cancer Biology and Pharmacology, Genome Institute of Singapore, Agency for Science, Technology and Research, 60 Biopolis Street \#02-01, Biopolis, Singapore 138672, Singapore. Tel: + 656478 9003; Fax: + 656478 9003; E-mail: yuq @ gis.a-star.edu.sg

Keywords: EZH2; apoptosis; DNA damage; checkpoint

Abbreviations: $E Z H 2$, enhancer of Zeste homologe 2; siRNA, small interfering RNA; ChIP, chromatin immunoprecipitation; H3K27me3, H3 with trimethylated lysine 27; DZNep, deazaneplanocin A; PARP, poly (ADP-ribose) polymerase; HEK293, human embryonic kidney 293

Received 15.12.10; revised 11.3.11; accepted 29.3.11; Edited by M Oren; published online 06.5.11
} 

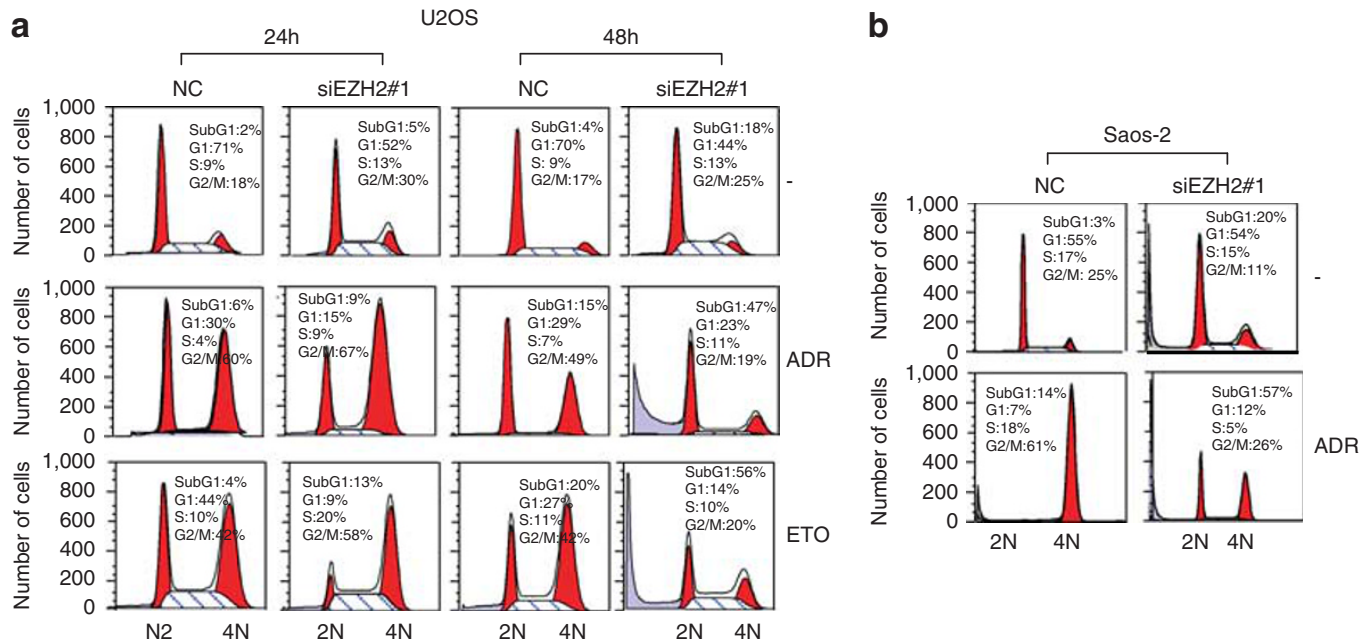

Figure 1 Depletion of EZH2 results in defective G1 and G2/M cell cycle checkpoints and promotes apoptosis in both p53 wild-type and deficient cancer cells. (a) U2OS cells transfected with NC or EZH2 siRNA were treated with adriamyxin (ADR, $1 \mu \mathrm{M})$ or etoposide $(\mathrm{ETO}, 10 \mu \mathrm{M})$ for 24 or $48 \mathrm{~h}$, and then were collected for flow cytometry analysis. (b) Saos-2 cells transfected with NC or EZH2 siRNA were treated with ADR $(1 \mu \mathrm{M})$ for $48 \mathrm{~h}$, and then were collected for flow cytometry analysis

both p53 wild-type and p53-deficient cells. Western blot analysis in U2OS and HCT116 cells revealed that EZH2 depletion largely abolished ADR- or ETO-induced p21 accumulation, but had no effect on p53 itself (Figure 2a and Supplementary Figure S1b) or expression of other p53 targets known to be involved in cell cycle arrest or apoptosis (Gadd45, Puma, Noxa or Pig3; Supplementary Figure S1c). EZH2 depletion also diminished DNA damage-induced Chk1 phosphorylation, but had no obvious effect on Chk2 phosphorylation (Figure 2a and Supplementary Figure S1b). Consistent with increased apoptosis and loss of DNA damage checkpoints in EZH2-depleted cells, we observed increased poly (ADP-ribose) polymerase (PARP) cleavage and $\mathrm{H} 2 \mathrm{AX}$ phosphorylation (Figure $2 \mathrm{a}$ and Supplementary Figure S1b). In p53-deficient Saos-2 HCT116 p53 ${ }^{-1-}$ and H1299 cells, attenuated Chk1 phosphorylation by DNA damage, accompanied by enhanced PARP cleavage and $\mathrm{H} 2 \mathrm{AX}$ phosphorylation, was also observed (Figure 2b, Supplementary Figures S2b and S2d). These effects of EZH2 on DNA damage-mediated p21 induction and Chk1 activation are in agreement with known roles of p21 and Chk1 in inducing G1 and G2/M checkpoints, respectively, and protecting DNA damage-induced apoptosis. 4,9,13,29

The specificity of EZH2 knockdown was further confirmed by another EZH2 small interfering RNA (siRNA\#2) that targets the $5^{\prime}$-untranslated region of EZH2 mRNA (Figure 2c). This siRNA is unable to silence the ectopic EZH2 and was thus used for the below rescue experiments. Indeed, the loss of induction of both p21 and p-Chk1 by ADR in EZH2depleted cells, as well as the corresponding changes in G1 arrest and apoptosis, was markedly restored by ectopic $\mathrm{EZH} 2$, but not by a deletion mutant lacking the catalytic SET domain $(\mathrm{EZH} 2 \Delta)$ that is required for histone H3K27me3 (Figures $2 d-f)$. These findings substantiate a crucial role of $\mathrm{EZH} 2$ in conferring cell cycle arrest rather than apoptosis during DNA damage in cancer cells, and this function of EZH2 requires the SET domain, which is essential for its gene silencing activity. ${ }^{22}$
Of note, p21 protein downregulation was not due to a decrease in p21 mRNA level (Figure $2 \mathrm{~g}$ ), and was restored upon treatment with proteasome inhibitors (Figure $2 \mathrm{~h}$ ). This indicates a post-translational regulation of p21 in this context, which involves a proteasome-dependent protein degradation mechanism. In contrast, the reduced Chk1 activation does not seem to be regulated by such a mechanism (Figure2h).

EZH2 depletion results in induction of FBXO32, a target directly suppressed by EZH2 in multiple human cancer cells. Given the well-known role of p21 in determining the cellular sensitivity to DNA-damaging agents ${ }^{3,4,7,9,13,14,30}$ and its potential post-translational regulation in the above EZH2 context, we sought to focus on p21 and to identify EZH2repressed genes that are potentially involved in regulating p21 protein turnover, and thereby affecting cellular response to DNA damage. We performed EZH2 knockdown in HCT116 and MCF7 cells, and identified 80 genes that were upregulated upon $\mathrm{EZH} 2$ depletion in both cell lines (Figure $3 \mathrm{a}$ and Supplementary Table S1; using threefold cutoff). To determine whether these potential EZH2 targets are also downregulated in clinical samples, we performed expression analysis of this gene set in 24 pairs of patientderived primary colon tumor samples and matched normal controls. In all, 12 out of 80 genes were found to be downregulated in tumor samples as compared with normal controls (Figure $3 b$ ). Among these clinically relevant EZH2 targets was $\mathrm{FBXO32}$, a member of F-box protein family, known to have a role in binding to and directing its protein substrates for proteasome-mediated degradation, ${ }^{31,32}$ whereas the other targets do not seem to have annotated roles in protein degradation. Moreover, $\mathrm{EZH} 2$ expression is inversely correlated with $F B X 032$ expression in the clinical samples, but not with its close family member $F B X O 31$ (Figure $3 c$ and Supplementary Table S2) or other F-box family members (data not shown), thereby indicating a selective repression of $F B X 032$ by $\mathrm{EZH} 2$. We have previously shown that $F B X 032$ is targeted by $\mathrm{PRC} 2$ 
a

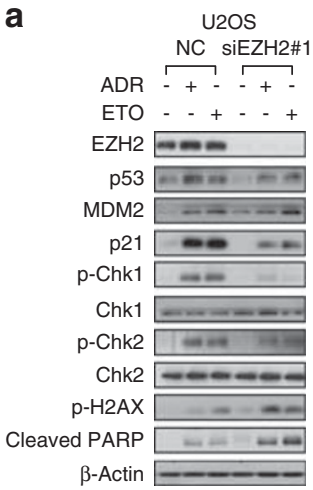

b

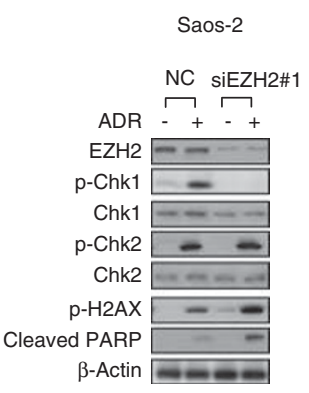

C

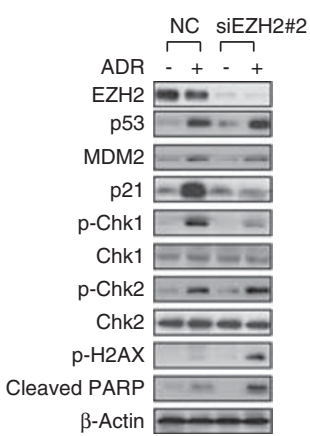

d

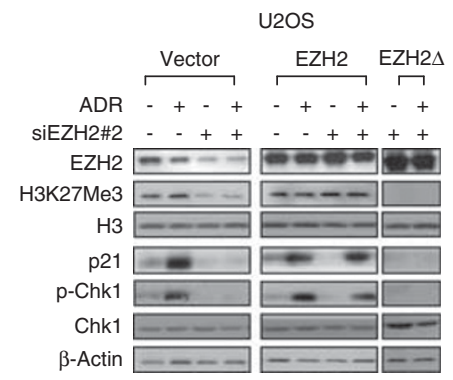

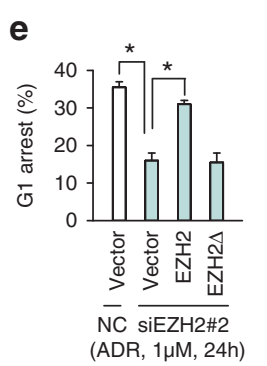

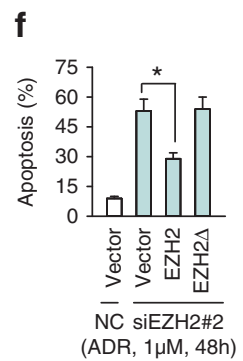

g

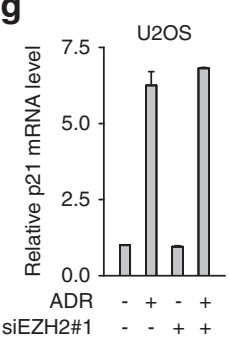

h

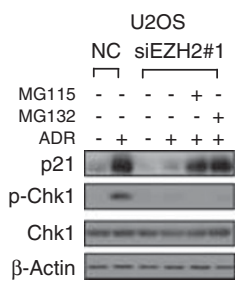

Figure 2 EZH2 depletion abolished p21 and Chk1 activation in response to DNA damage. (a) Western blot analysis showing that EZH2 depletion abolished ADR (1 $\mu$ M)or ETO $(10 \mu \mathrm{M})$-induced p21 accumulation and Chk1 phosphorylation, and increased levels of phosphorylated H2AX and PARP cleavage. $\beta$-Actin is shown as loading control. (b) Western blot analysis in Saos-2 cells showing that EZH2 depletion abrogated ADR-induced Chk1 phosphorylation, and increased levels of phosphorylated H2AX and PARP cleavage. $\beta$-Actin is shown as loading control. (c) Western blot analysis showing the effects of another EZH2 siRNA (\#2) targeting $5^{\prime}$-untranslated region of EZH2 mRNA on cell cycle checkpoint proteins. (d) Western blot analysis showing the rescue effects of EZH2 and EZH2 SET domain deletion (EZH2 $\Delta$ ) on p21 and Chk1. FACS analysis in U2OS showing the effects of EZH2 depletion on checkpoint abrogation (e) and apoptosis (f) are rescued by wild-type EZH2, but no by EZH2 $\Delta$. Data are means \pm S.D. $(n=3),{ }^{*} P<0.05$ (Student's t-test). (g) Quantitative real-time PCR (qRT-PCR) analysis of p21 mRNA levels in NC- or siEZH2-treated U2OS cells in the presence or absence of ADR $(1 \mu \mathrm{M})$ treatment for $24 \mathrm{~h}$. Data are means \pm S.D. $(n=3)$. (h) Western blot analysis of p21 and Chk1 in U2OS cells treated with ADR and EZH2 siRNA in the presence or absence of proteasome inhibitors MG132 $(5 \mu \mathrm{M})$ or MG115 $(10 \mu \mathrm{M})$

complex in breast cancer cells and contributes to histone methylation inhibitor deazaneplanocin A (DZNep)-induced apoptosis. ${ }^{33}$ In addition, FBXO31 is known to have a role in modulating DNA damage response by inducing cyclin D1 degradation. Thus, it seemed possible that the induction of FBXO32 upon EZH2 knockdown is responsible for the p21 protein degradation in DNA damage response.

Before exploring a potential role of $\mathrm{FBXO} 2$ in p21 regulation, we first wanted to validate whether $\mathrm{FBXO} 32$ is directly repressed by EZH2 in multiple cancer cells. Quantitative real-time PCR and western blot analysis confirmed a selective induction of $F B X O 32$ (but not $F B X 031$ ) upon $\mathrm{EZH} 2$ knockdown in multiple human cancer cell lines (Figures $3 d$ and e). Chromatin immunoprecipitation (ChIP) assay shows that $\mathrm{EZH} 2$ and $\mathrm{H} 3 \mathrm{~K} 27 \mathrm{me} 3$ were markedly enriched in the FBXO32 promoter in HCT116 (Figure 3f), U2OS and MCF7 cells, but not in non-cancerous MCF10A cells (Figure $3 \mathrm{~g}$ ), and $\mathrm{EZH} 2$ knockdown resulted in reduced $\mathrm{EZH} 2$ and H3K27me3 enrichment at $F B X O 32$ promoter (Figure 3h), demonstrating a direct repression of $\mathrm{FBXO} 32$ by $\mathrm{EZH} 2$ in cancer cells, but not in non-transformed cells. Accordingly, an inverse correlation between $E Z H 2$ and $F B X O 32$ expression was observed in MCF7 and MCF10A cells (Figure 3i). Collectively, these findings identify $\mathrm{FBXO} 32$ as a bona fide direct target of $\mathrm{EZH} 2$ in multiple human cancers.
Induction of $\mathrm{FBXO32}$ following $\mathrm{EZH} 2$ depletion is required for p21 degradation during DNA damage. To validate a hypothetical role of $\mathrm{FBXO} 32$ in $\mathrm{p} 21$ protein regulation, we performed single or double knockdown of EZH2 and/or FBXO32 in U2OS, HCT116 and MCF7 cells. In U2OS cells, diminished p21 resulting from EZH2 knockdown during DNA damage was substantially restored by concomitant knockdown of FBXO32 (Figure 4a), which was accompanied by a marked rescue of $\mathrm{G} 1$ arrest (Supplementary Figure S3) and apoptosis (Figure 4b). Downregulation of $\mathrm{p}$-Chk1, however, was not restored by concomitant FBXO32 knockdown, indicating a selective effect of FBXO32 toward p21 but not Chk1. A similar result was also found in HCT116 and MCF7 cells in which FBXO32 was knocked down by an shRNA targeting a different sequence (Figures $4 \mathrm{c}-\mathrm{e}$ ). These findings revealed a crucial role of FBXO32 in p21 downregulation following EZH2 knockdown and DNA damage in cancer cells. In noncancerous MCF10A cells where FBXO32 is not silenced, $\mathrm{EZH} 2$ knockdown did not further increase FBXO32 expression, and thus did not affect ADR-induced p21 induction (Figure 4e). Thus, the effect of EZH2 knockdown on FBXO32 induction and subsequent abolishment of p21 protein accumulation during DNA damage response seemed to be cancer specific. 


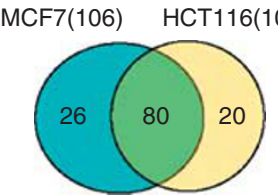

b

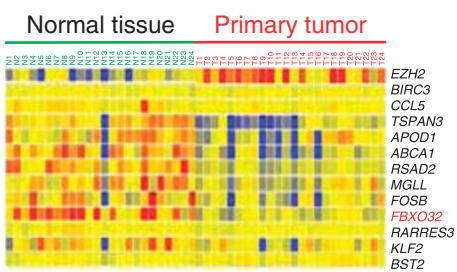

d

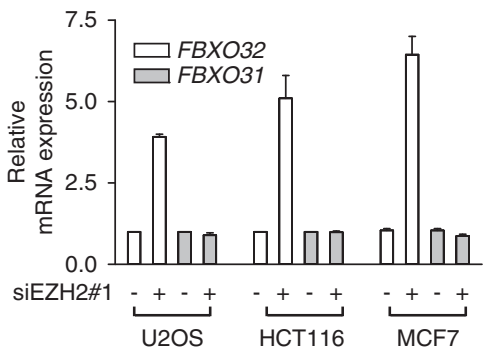

g

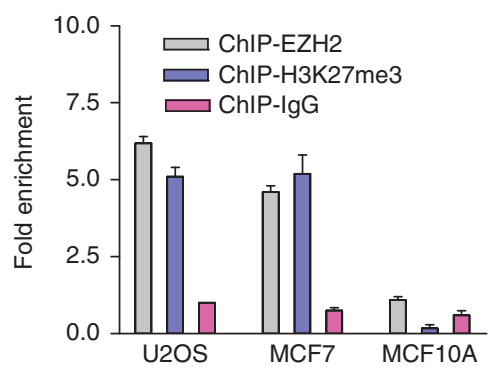

C
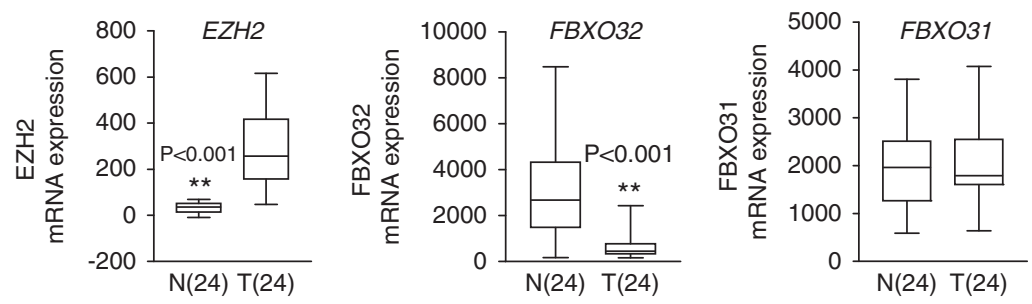

e

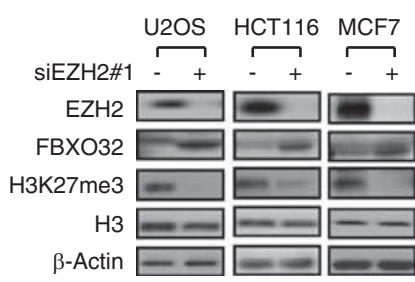

f

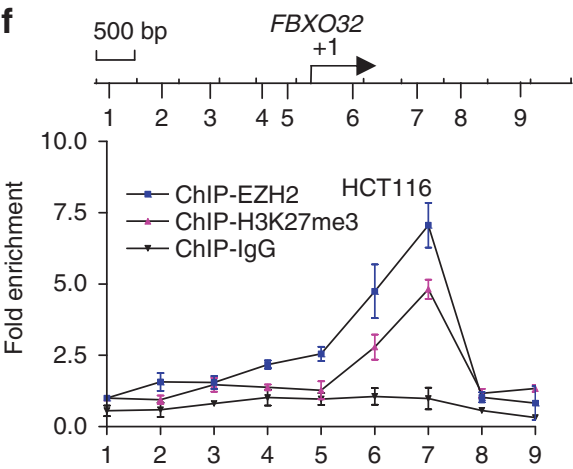

i

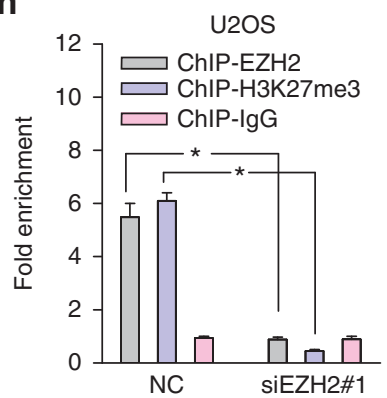

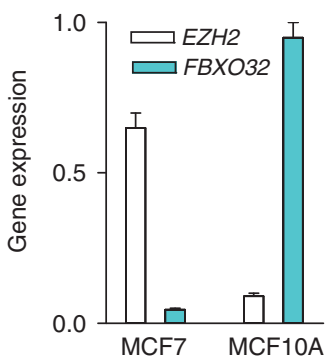

Figure $3 \mathrm{EZH} 2$ directly represses FBXO32 expression in multiple cancer cells. (a) Vent diagram showing the overlapping genes upregulated upon EZH2 knockdown in U2OS and HCT116 cells. (b) Hierarchical clustering of EZH2 target genes that downregulated in 24 pairs of patient-derived primary colon tumor samples and matched normal controls. (c) Box plots showing the mRNA expression levels of EZH2, FBXO32 and FBXO31 in 24 pairs of patient-derived primary colon tumor samples and matched normal controls. (d) Quantitative real-time PCR (qRT-PCR) analysis of FBXO32 and FBXO31 mRNAs in U2OS, HCT116 and MCF7 cells treated with EZH2 siRNA. (e) Western blot analysis of FBXO32 protein levels in U2OS, HCT116 and MCF7 cells treated with EZH2 siRNA. (f) ChIP-PCR analysis showing the EZH2 and H3K27me3 enrichments at FBXO32 locus in HCT116 cells. (g) ChIP-PCR showing EZH2 and H3K27me3 enrichments at FBXO32 locus in U2OS and MCF7 cells, but not in MCF10A cells. (h) ChIPPCR showing EZH2 and H3K27me3 enrichments at FBXO32 locus in U2OS treated with NC or EZH2 siRNA. (i) qRT-PCR analysis of EZH2 and FBXO32 mRNAs in MCF7 and MCF10A cells

We next evaluated whether ectopic expression of $\mathrm{FBXO} 32$ would mimic the effects of EZH2 knockdown on p21, G1 arrest and apoptosis. Overexpression of FBXO32 in HCT116 cells resulted in inhibition of p21 induction by ETO and ADR, orchestrated by increased PARP cleavage, but had no effect on p-Chk1 (Figure 4f). FBXO32-overexpressing cells synchronized in mitosis with nocodazole $(400 \mathrm{nM})$, which blocks exit from mitosis, showed reduced arrest in $\mathrm{G} 1$ phase after ADR treatment as compared with vector control cells (Figure 4g). As expected, FBXO32-overexpressing HCT116 cells were much more sensitive to ADR- or ETO-induced apoptosis (Figure 4h). By contrast, FBXO32 overexpression

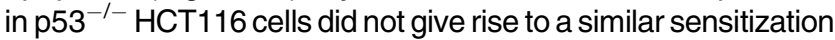
to ADR or ETO (Figure 4h). Moreover, consistent with a role of FBXO32 in downregulating p21, HCT116 cells depleted of p21 by shRNA were much more sensitive to ADR or ETO treatment, and ectopic expression of FBXO32 in these cells did not further increase the magnitude of apoptotic response to ADR or ETO (Figure 4h). In summary, these data suggest that FBXO32induced p21 downregulation is a key functional target of EZH2 knockdown, which has a crucial role in causing p53 wild-type cancer cell fate switch in response to DNA damage. 
a

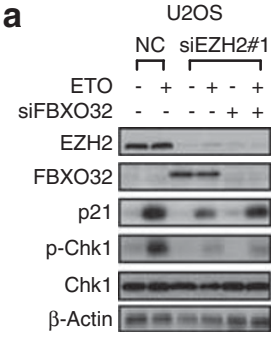

f b

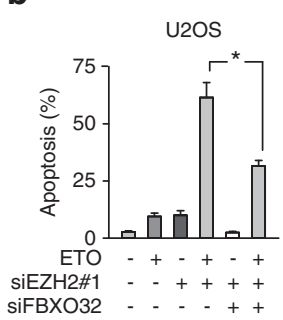

C

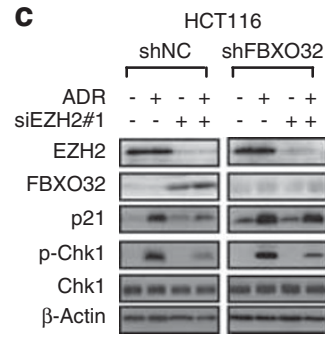

d

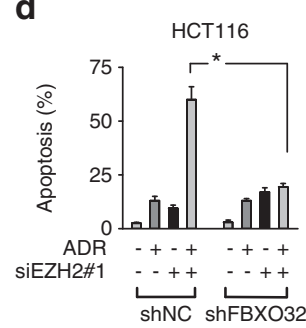

e

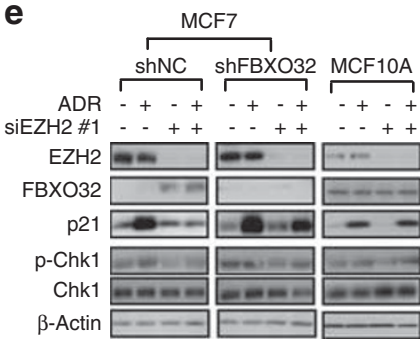

g

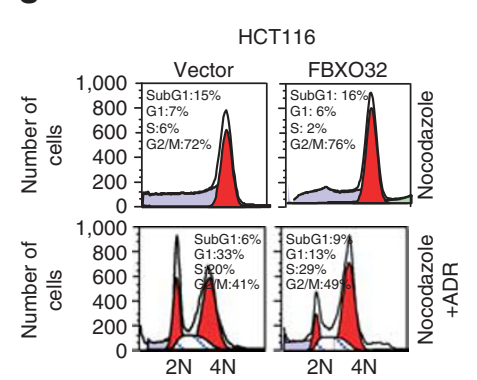

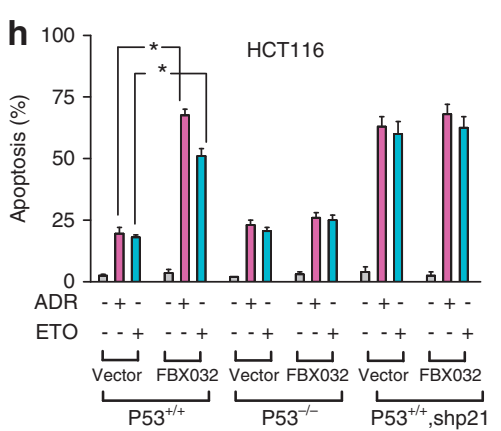

Figure 4 Induction of FBXO32 following EZH2 depletion is functionally required for p21 degradation and increased apoptosis during DNA damage in p53 wild-type cells. (a) Western blot analysis of indicated proteins in ETO-treated U2OS cells depleted of EZH2, FBXO32 or both by respective siRNAs. ETO, $10 \mu \mathrm{M}$ for $24 \mathrm{~h}$. (b) Cells were treated as a for $48 \mathrm{~h}$, and apoptosis was assessed by FACS analysis. (c) Western blot analysis of indicated proteins in ADR-treated HCT116 cells expressing a FBXO32 shRNA, with or without EZH2 siRNA. (d) Apoptosis analysis of HCT116 cells treated as in c. (e) Western blot analysis of indicated proteins in MCF7 expressing a FBXO32 shRNA and MCF10A cells treated with ADR and EZH2 siRNA. (f) Western blot analysis showing the indicated proteins in HCT116 cells overexpressing FBXO32 or a control vector treated with ADR or ETO (g) FACS analysis of HCT116 cells expressing FBXO32 or a control vector that were synchronized in mitosis with nocodazole (400 nM), followed by treatment with ADR for $10 \mathrm{~h}$. (h) Apoptosis analysis of $\mathrm{HCT} 116 \mathrm{p} 53^{+/+}, \mathrm{HCT} 116 \mathrm{p} 53^{-/-}$or HCT116 expressing shp21 that were infected with retroviruses expressing FBXO32 or a control vector and treated with ADR or ETO for $48 \mathrm{~h}$

FBX032 interacts with p21 to induce p21 protein degradation in the F-Box and ubiquitin-independent manner. F-box proteins are known to induce substrate degradation through protein-protein interaction. ${ }^{31} \mathrm{We}$ therefore investigated a potential interaction between FBXO32 and p21 by coimmunoprecipitation assay. Myctagged FBXO32 and/or V5-tagged p21 were transiently expressed in human embryonic kidney 293 (HEK293) and HCT116 cells in the absence or presence of proteasome inhibitor MG132. As expected, coexpression of FBXO32 caused a downregulation of exogenous p21, which was restored in the presence of MG132 (Figure 5a). We detected a clear interaction between exogenous FBXO32 and p21, which was further enhanced by MG132 (Figure 5a) In addition, we detected an endogenous interaction between the two proteins in ADR-treated HCT116 cells after EZH2 knockdown in the presence of MG132 (Figure 5b). These data demonstrate that FBXO32 physiologically interacts with p21 and induces p21 degradation. To identify the functional domain of FBXO32 required for p21 degradation, we performed a series of $\mathrm{FBXO32-depletion} \mathrm{experiment}$ analysis (Figure 5c). Unexpectedly, EZH2 mutant lacking the $\mathrm{F}$-box (FBXO32 $\mathrm{F}$ ) remained active in causing p21 degradation (Figure $5 \mathrm{c}$ ), suggesting that the F-Box is not required for p21 degradation. Moreover, unlike the C-terminal deletion mutant $\Delta \mathrm{C}$ (272-355), which was able to induce p21 degradation, FBXO32 lacking the N-terminal domain (1-50) was unable to induce p21 degradation (Figure $5 \mathrm{c}$ ), suggesting an important role of $\mathrm{N}$-terminal domain (1-50) in p21 degradation. Consistent with this finding, we found that $\mathrm{FBXO} 32 \Delta \mathrm{F}$ remained to interact with p21, whereas FBXO32 $\Delta \mathrm{N}(1-50)$ was unable to bind to p21 (Figure $5 \mathrm{~d}$ ). Consistent with a lack of F-box involvement in p21 degradation, we found that ectopic FBXO32 expression did not result in ubiquitination of $\mathrm{p} 21$, as assessed via coexpression of hemaglutinin (HA)-tagged ubiquitin (Supplementary Figure S4), nor did we detect Skp1 and Cul1, the components of SCF ubiquitin ligase in the FBXO32-p21 complex (data not shown). Thus, FBXO32 induces p21 degradation via an ubiquitinindependent but proteasome-dependent mechanism; such a mechanism has been previously described for p21 degradation in other contexts. ${ }^{34,35}$ In agreement with a crucial role of $\mathrm{N}$ terminal domain (1-50) of $\mathrm{FBXO} 32$ in p21 degradation, FBXO32 $\Delta N(1-50)$ was unable to abolish ETO-induced p21 induction and did not promote apoptosis induction by ETO or ADR, whereas FBXO32 $\Delta F$ and $F B X 032 \Delta C(272-355)$ retained that function (Figures $5 e$ and $f$ ). Taken together, these findings demonstrate that the interaction between FBXO32 and p21, which is mediated by its N-terminus, but not the F-box, is critical for p21 degradation in an ubiquitin-independent manner.

Pharmacological depletion of EZH2 phenocopies EZH2 knockdown in modulating DNA damage response. Thus, so far, we have demonstrated an epigenetic mechanism of $\mathrm{EZH} 2$ in regulating cellular response to DNA damage induced by genotoxic agents. We next tested whether this finding can be translated into a viable treatment strategy to improve chemotherapeutic response. We have previously 
a

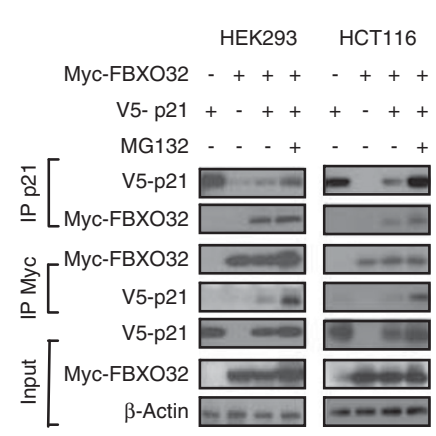

b

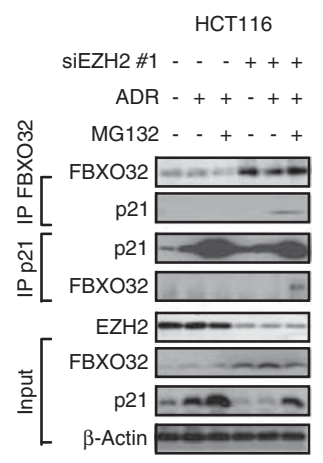

C
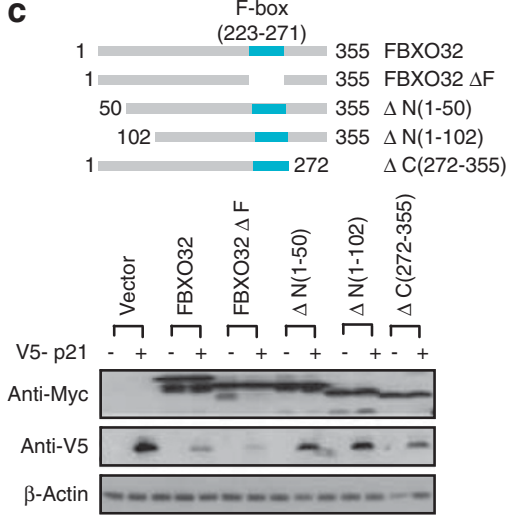

d

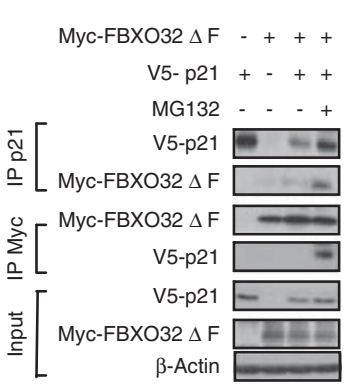

HEK293 e

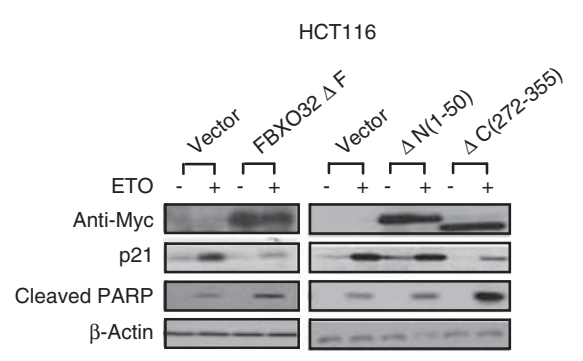

f

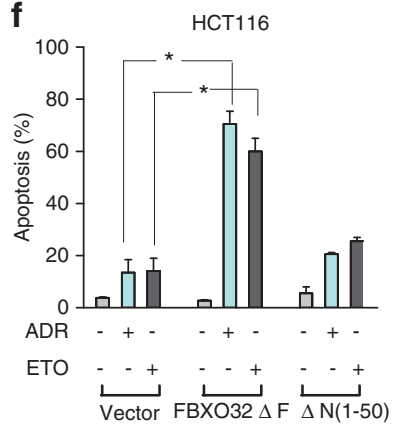

Figure 5 FBXO32 interacts with and induces p21 protein degradation in F-box-independent manner. (a) Coimmunoprecipitation of FBXO32 with p21 in HEK293 and HCT116 cells. Myc-tagged FBXO32 and/or V5-tagged p21 were transiently expressed in HEK293 or HCT116 cells and incubated with or without proteasome inhibitor MG132 $(5 \mu \mathrm{M})$ for $6 \mathrm{~h}$ to prevent p21 degradation. Immunoprecipitates were performed using indicated antibodies to detect interactions. (b) Coimmunoprecipitation of endogenous FBXO32 and p21 in HCT116 cells. HCT116 cells were transfected with siEZH2 and followed by ADR ( $1 \mu \mathrm{M}, 24 \mathrm{~h})$ treatment. Cells were treated with MG132 $(5 \mu \mathrm{M})$ for $6 \mathrm{~h}$ before harvesting (c) Schematic representation of various FBXO32 deletion mutants (upper panel). Western blot analysis of the effect of FBXO32 deletion mutants on p21 degradation in HEK293 cells (bottom panel). (d) Myc-tagged FBXO32 deletion mutants (FBXO32 $\mathrm{F}$ or FBXO32 $\Delta \mathrm{N}(1-50)$ ) and/or V5-tagged p21 were transiently expressed in HEK 293 cells and incubated with or without MG132 $(5 \mu \mathrm{M})$ for $6 \mathrm{~h}$ before harvesting for immunoprecipitation and western blot analysis. (e) Western blot analysis of indicated proteins in HCT116 cells expressing FBXO32 $\mathrm{F}$, FBXO32 $\mathrm{N}(1-50)$ or FBXO32 $\Delta \mathrm{C}(272-355)$ in response to ETO treatment for $24 \mathrm{~h}$. (f) Apoptosis analysis of HCT116 cells expressing FBXO32 $\mathrm{F}$, or FBXO32 $\mathrm{N}(1-50)$ in response to $\mathrm{ADR}$ or ETO treatment for $48 \mathrm{~h}$

shown that histone methylation inhibitor DZNep can deplete $\mathrm{EZH} 2$ complex, resulting in reactivation of genes suppressed by $\mathrm{EZH} 2$ in cancer cells. ${ }^{33}$ Therefore, we investigated whether DZNep treatment would mimic EZH2 knockdown and give rise to similar effects. As noted in Figure 6, in both p53-proficient U2OS and HCT116 cells and p53-deficient Saos-2 and H1299 cells, pretreatment with DZNep converted ADR- or ETO-induced cell cycle arrest to marked apoptosis (Figures 6a and d and Supplementary Figures S5a, S5c and S5e). Similar to EZH2 knockdown, EZH2 depletion by DZNep in cancer cells resulted in FBXO32 induction, largely abolished p21 and/or p-Chk1 induction by ADR or ETO, increased PARP cleavage and H2AX phosphorylation (Figures $6 \mathrm{~b}$ and e and Supplementary Figures S5b, S5d and $\mathrm{S} 5 \mathrm{f})$, and reduced $\mathrm{H} 3 \mathrm{~K} 27 \mathrm{me} 3$ at $\mathrm{FBXO} 32$ promoter (Figure 6c), but did not affect other p53 target genes (Supplementary Figure S5g). In contrast, DZNep did not promote ADR- or ETO-induced apoptosis in non-transformed MCF10A, IMR90 and RWPE cells (data not shown). Again, DZNep-induced p21 protein downregulation was not a result of decreased p21 mRNA (Supplementary Figure S5g), and was also prevented by MG132 treatment (Figure 6f). Importantly, this effect of DZNep in HCT116 cells was largely abolished when FBXO32 was knockdown (Figures
$6 \mathrm{~g}$ and $\mathrm{h}$ ), further confirming a crucial role of FBXO32 in DNA damage-induced apoptosis following EZH2 depletion. Thus, pharmacological depletion of EZH2 by DZNep phenocopied the effects of genetic knockdown of EZH2 on FBXO32, p21, cell cycle checkpoints and apoptosis in DNA damage response. Although DZNep may not be a specific EZH2 inhibitor, it provides a proof of principle that specific smallmolecule inhibitor of $\mathrm{EZH} 2$, when available, may provide therapeutic benefits if combined with standard chemotherapy for cancer patients carrying $\mathrm{EZH} 2$ overexpression.

\section{Discussion}

Our data provide the first demonstration that EZH2-mediated gene silencing in cancer cells has an important role in determining the cellular outcome in response to DNA damage. A striking finding is that inhibition of $\mathrm{EZH} 2$ abrogates both $\mathrm{G} 1$ and G2/M checkpoints and promotes DNA-damaging agentinduced apoptosis in both p53 wild-type and p53-deficient cancer cells (Figure 6i). Thus, these findings bear important implications for cancer treatment and indicate that therapeutic inhibition of EZH2 might appeal as an attractive approach to sensitize standard chemotherapy for cancers that overexpress $\mathrm{EZH} 2$. These findings point to a potential application of 
a

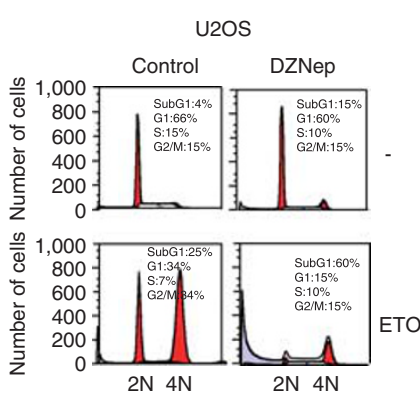

e

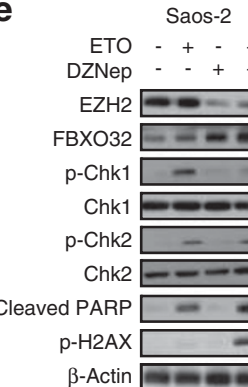

b

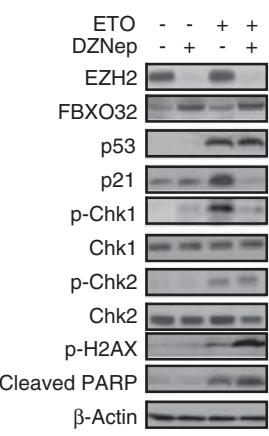

C

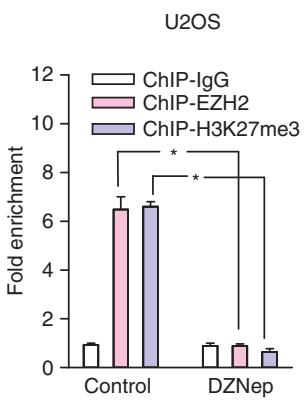

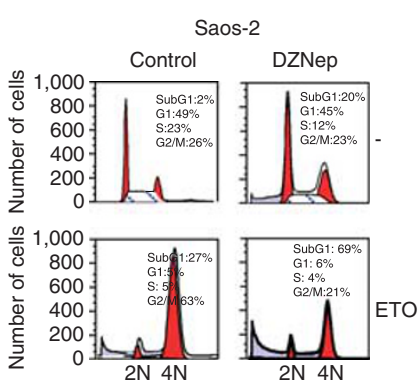

g

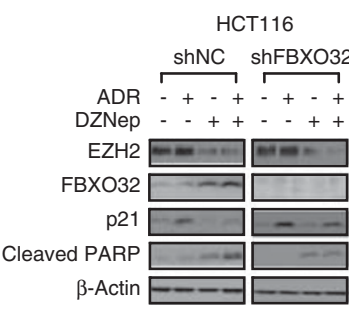

h i

Figure 6 Pharmacological depletion of EZH2 phenocopies EZH2 knockdown in modulating DNA damage response. (a) FACS analysis of U2OS cells treated with ETO, DZNep or both. Cells were treated with DZNep $(5 \mu \mathrm{M})$ for $24 \mathrm{~h}$, followed by ETO $(10 \mu \mathrm{M})$ for $48 \mathrm{~h}$. (b) Western blot analysis of indicted proteins in cells treated as a. (c) ChIP analysis of EZH2 and H3K27me3 enrichment at FBXO32 promoter in DZNep-treated or -untreated cells. (d) FACS analysis of Saos-2 cells treated with ETO, DZNep or both. Cells were treated with DZNep $(5 \mu \mathrm{M})$ for $24 \mathrm{~h}$, followed by ETO $(10 \mu \mathrm{M})$ for $48 \mathrm{~h}$. (e) Western blot analysis of indicted proteins in cells treated as c. (f) Western blot analysis of indicated proteins in HCT116 cells treated with DZNep or ADR or both in the presence of MG132. (g) Western blot analysis of indicated proteins in HCT116 cells expressing a FBXO32 shRNA in response to ADR, DZNep or both. (h) Apoptosis analysis of cells treated in $\mathbf{g}$. (i) A proposed model for a role of EZH2 in modulating DNA damage response

$\mathrm{EZH} 2$ inhibitors that are under active development by pharmaceutical companies.

Mechanistically, this effect of EZH2 on p53 wild-type cancer cells is mainly mediated through suppression of $\mathrm{FBXO32-}$ directed p21 degradation to maintain the cell cycle arrest rather than apoptosis in response to DNA damage. Also, p21 level is well known to be crucial in determining cellular sensitivity to DNA-damaging agents. . $^{3,8,13,36,37}$ Therefore, manipulation of p21 level seems to be a feasible approach for modulating chemotherapeutic response. ${ }^{30}$ For example, pharmacological inhibition of p21 protein translation using mTOR inhibitor RAD001 has been shown to be effective in converting DNA damage-induced p53 response from growth arrest to apoptosis. ${ }^{14}$ Hence, identification of EZH2-mediated FBXO32 repression in DNA damage-induced cell cycle checkpoint control through regulation of p21 stability suggests an epigenetic mechanism, regulating DNA damage response that can be targeted to augment chemotherapeutic response. Importantly, repression of $\mathrm{FBXO} 32$ by $\mathrm{EZH} 2$ is cancer specific, as such, therapeutic targeting $E Z H 2$ that is often overexpressed in human cancer is expected to result in selective sensitization in cancer cells, with a minimum effect on normal cells.

In addition to the effect on p21, EZH2 knockdown also abolishes Chk1 phosphorylation in response to DNA damage. Genetic or small-molecule inhibition of Chk1 phosphorylation is known to induce chemosensitization selectively in p53-mutant cancer cells. ${ }^{5,29,38-40}$ This is correlated with the abolished G2/M arrest and increased apoptosis in p53deficient cancer cells upon EZH2 knockdown. However, this effect of EZH2 on Chk1 is not related to FBXO32, as neither FBXO32 knockdown nor overexpression has any effect on Chk1 phosphorylation. So, the regulation of EZH2 on Chk1 appears to be mediated through a distinct mechanism. Further investigation of the molecular mechanisms is likely to provide additional insights into epigenetic regulation of DNA damage response.

Given the toxic side effect of chemotherapy, any sensitizer that can direct even a mild DNA damage response toward an apoptotic program would have the potential to enhance the efficacy of DNA-damaging chemotherapeutic agents and reduce the toxic effects. ${ }^{41,42}$ As EZH2 depletion simultaneously abolishes both DNA damage-induced G1 and G2/M checkpoints, therapeutic targeting $\mathrm{EZH} 2$, as demonstrated here as a proof of concept using DZNep, in combination with standard chemotherapy, may provide a unique advantage over the existing approaches for treatment of cancer patients carrying wild-type or mutant p53. Furthermore, given a potential role of $\mathrm{EZH} 2$ or PRC2 in maintenance of cancer stem cells ${ }^{43,44}$ and a recent finding showing that DNA damage-activated p21 is required for self-renewal of leukemia stem cells, ${ }^{45} \mathrm{EZH} 2$ inhibition might also have the potential for overcoming chemoresistance phenotype typically seen in cancer stem cells, and thereby preventing tumor recurrence after chemotherapy. ${ }^{46}$ 


\section{Materials and Methods}

Cell culture and drugs. The human colorectal cancer HCT116 cells and its derived isogenic $p 53^{-1-}$ HCT116 cells were kindly provided by Dr. Bert Vogelstein (John Hopkins University, MD, USA). Other cell lines used in this study, including human osteosarcoma U2OS and Saos-2, lung caner H1299, breast cancer MCF7 and HEK epithelial 293 cells, were obtained from the American Type Culture Collection (ATCC, Manassas, VA, USA) and maintained in Dulbecco's modified Eagle's medium supplemented with $10 \%$ fetal bovine serum and antibiotics in a $37^{\circ} \mathrm{C}$ humidified incubator containing $5 \% \mathrm{CO}_{2}$. Human breast epithelial MCF10A cells were obtained from ATCC and maintained, as recommended. ${ }^{47}$ adramycin (ADR), etoposide (ETO), nocodazole (Noco), proteasomal inhibitors MG132 and MG115 were purchased from Sigma-Aldrich (St. Louis, MO, USA).

Plasmids and stable cell lines. pcDNA4/FBXO32-Myc was generated by real-time PCR using total RNA from normal colon tissue. Retroviral-mediated gene transfer was performed using pMN-GFP/IRES retrovirus vector-expressing FBXO32. Infected cells were sorted by GFP signals and expanded for in vitro studies. EZH2 wild-type and SET domain deletion mutant (EZH2 $\Delta$ ) plasmids and HA-tagged ubiquitin expression plasmid have been described previously, respectively. ${ }^{28,48}$ The full-length human $\mathrm{FBXO32}$ was used as a template to generate FBXO32 deletion mutations. All constructs were confirmed by sequencing.

RNA interference. Specific siRNA oligos targeting EZH2 and FBXO32 mRNAs were described previously. ${ }^{28,33}$ The non-targeting control was purchased from Dharmacon (Lafayette, CO, USA). For EZH2 knockdown, cells were sequentially transfected two times at $24 \mathrm{~h}$ interval to secure efficient gene silencing. Cells were transfected with $100 \mathrm{nM}$ final concentration of siRNA duplexes using Lipofectamine RNAiMax (Invitrogen, Carlsbad, CA, USA) following the manufacturer's instructions. To generate FBXO32 shRNA stable cell lines, siRNA oligos targeting FBXO32 (sequence: CAGAAGATTATATGGCGCGAA) were cloned into the PSIREN-RetroQ retroviral expression vector (BD Bioscences, Rockville, MD, USA) according to the manufacturer's instruction. Virally infected cells were selected in a medium containing $2 \mu \mathrm{g} / \mathrm{ml}$ puromycin. Individual drug-resistant clones were collected and expanded.

Immunoblot analysis and coimmunoprecipitation. Protein extracts were prepared by lysis in RIPA buffer containing ( $50 \mathrm{mM}$ Tris-HCl, pH 7.4, $150 \mathrm{mM}$ $\mathrm{NaCl}, 1 \%$ Nonidet P-40, $0.5 \%$ sodium deoxycholate, $0.1 \%$ SDS, $1 \mathrm{mM}$ EDTA, $50 \mathrm{mM} \mathrm{NaF}, 0.1 \mathrm{mM} \mathrm{Na}_{3} \mathrm{VO}_{4}$ ) and protease inhibitor cocktail (Roche, Indianapolis, IN, USA). Lysates were resolved by SDS-PAGE, transferred onto an immobilon membrane (Millipore, Billerica, MA, USA) and probed with indicated antibodies. For coimmunoprecipitation experiments, cells were lysed and incubated with the indicated antibodies and protein G-sepharose beads overnight at $4^{\circ} \mathrm{C}$. Beads were washed four times with lysis buffer. The bound proteins were dissolved in SDS sample buffer, resolved by SDS-PAGE and immunoblotted with the indicated antibodies. Where indicated, proteasome inhibitors MG132 $(5 \mu \mathrm{M})$ or MG115 $(10 \mu \mathrm{M})$ were used to treat cells for $8 \mathrm{~h}$ before protein extraction. Antibodies used in this study include: anti-EZH2 (Cell Signaling, Danvers, MA, USA), anti-p21 (Santa Cruz, Santa Cruz, CA, USA), anti-FBXO32 (Santa Cruz), anti-p53 (Santa Cruz), anti-MDM2 (Santa Cruz), anti-phospho-Chk1 (Cell signaling), anti-Chk1 (Santa Cruz), anti-phospho-Chk2 (Cell signaling), anti-Chk2 (Upstate, Lake Placid, NY, USA), anti-H3 (Cell signaling), anti-PARP (Cell Signaling), anti-H3K27me3 (Upstate), anti-V5 (Invitrogen), anti-Myc mouse monoclonal (Roche), anti-H2AX (Upstate), anti-HA mouse monoclonal (Santa Cruz), anti-SKP1 mouse monoclonal (Cell Signaling), anti-CUL1 rabbit polyclonal (Cell Signaling) anti-actin mouse monoclonal (Sigma, St. Louis, MO, USA).

Microarray gene expression analysis and Taqman assay. Total RNA was isolated using Trizol (Invitrogen) and purified with the RNeasy Mini Kit (Qiagen, Hilden, Germany). Reverse transcription was performed using an RNA Amplification kit (Ambion, Carlsbad, CA, USA). The microarray hybridization was performed using the Illumina Gene Expression Sentrix BeadChip HumanRef-8_V2 (Illumina, San Diego, CA, USA), and data analysis was performed using GeneSpring software from Agilent Technologies (Santa Clara, CA, USA), as described. $^{49}$ Quantitative real-time PCR was assessed using the PRISM 7900 Sequence Detection System (Applied Biosystems, Carlsbad, CA, USA) with specific probes from Applied Biosystems. Samples were normalized to the levels of glyceraldehyde-3-phosphate dehydrogenase mRNA.
Flow cytometry and cell cycle analysis. Cells were harvested and fixed in $70 \%$ ethanol. Fixed cells were stained with propidium iodide $(50 \mu \mathrm{g} / \mathrm{ml})$ after treatment with RNase $(100 \mu \mathrm{g} / \mathrm{ml})$. The stained cells were analyzed for DNA content by fluorescence-activated cell sorting (FACS) in a FACS Calibur (Becton Dickinson Instrument, San Jose, CA, USA). Cell cycle fractions were quantified using the CellQuest software (Becton Dickinson). For synchronization experiment, HCT116 cells expressing FBXO32 or empty vector were treated with Noco $(400 \mathrm{ng} / \mathrm{ml})$ for $16 \mathrm{~h}$, mitotically arrested cells were treated with ADR and then subjected to FACS for cell cycle analysis.

In vivo ubiquitination assay. In vivo ubiquitination was conducted, as described previously. ${ }^{50}$ Briefly, HEK293T cells were transfected with HA-ubiquitin, V5-tagged p21 and Myc-tagged FBXO32- or FBXO32 $\Delta F$-expressing plasmids using Fugene HD (Roche). Before harvest, cells were treated with MG132 (5 $\mu \mathrm{M})$ for $8 \mathrm{~h}$. Cells were lysed and polyubiquitinated p21 was detected by immunoprecipitation of HA-tagged ubiquitin followed by immunoblotting for p21.

ChIP assay. ChIP assays were performed, as described previously. ${ }^{49}$ Briefly, sonicated extracts were precleared and incubated with antibodies specific to either EZH2 (Active motif, Carlsbad, CA, USA), H3K27me3 (Upstate) or IgG control (sc-2027, Santa Cruz) at $4^{\circ} \mathrm{C}$ overnight on a $360^{\circ} \mathrm{C}$ rotator. The immunoprecipitated DNA was quantitated by quantitative real-time PCR using Applied Biosystems $7900 \mathrm{HT}$ Fast Real-Time PCR System (Applied Biosystems). The enrichment of $\mathrm{EZH} 2$ or $\mathrm{H} 3 \mathrm{~K} 27 \mathrm{me} 3$ binding at the examined regions was quantitated relative to the input amount. The sequences of the primers are shown in Supplementary Table S3.

Statistical analysis. The Student's $t$-test was done to assess the difference of cell death between various treatments with ADR or ETO treatment. $P<0.05$ was defined as the statistic significance.

\section{Conflict of interest}

The authors declare no conflict of interest.

Acknowledgements. This work was supported by the Agency for Science, Technology and Research of Singapore. We thank the Singapore Tissue Network for providing the human samples. STL is supported by the NUS Graduate School for Integrative Sciences and Engineering (NGS) scholarship.

\section{Author Contributions}

ZW and QY conceived the work, designed the experiments and wrote the manuscript. ZW performed all the knockdown and phenotype analysis, with technical assistance from XJ, YJL and JT, CL for FACS analysis and western blot analysis. STL, YQ and PLL performed FBXO32 constructs and immunoprecipitation analysis. MA performed the array analysis and RT-PCR. ZL constructed FBXO32 overexpressing stable cell lines.

1. Zhou BB, Elledge SJ. The DNA damage response: putting checkpoints in perspective. Nature 2000; 408: 433-439.

2. Sancar A, Lindsey-Boltz LA, Unsal-Kacmaz K, Linn S. Molecular mechanisms of mammalian DNA repair and the DNA damage checkpoints. Annu Rev Biochem 2004; 73: 39-85.

3. Fan S, Chang JK, Smith ML, Duba D, Fornace Jr AJ, O'Connor PM. Cells lacking CIP1/ WAF1 genes exhibit preferential sensitivity to cisplatin and nitrogen mustard. Oncogene 1997; 14: 2127-2136.

4. Li J, Tan J, Zhuang L, Banerjee B, Yang X, Chau JF et al. Ribosomal protein S27-like, a p53-inducible modulator of cell fate in response to genotoxic stress. Cancer Res 2007; 67: $11317-11326$.

5. Mukhopadhyay UK, Senderowicz AM, Ferbeyre G. RNA silencing of checkpoint regulators sensitizes p53-defective prostate cancer cells to chemotherapy while sparing normal cells. Cancer Res 2005; 65: 2872-2881.

6. Jascur T, Brickner H, Salles-Passador I, Barbier V, El Khissiin A, Smith B et al. Regulation of p21(WAF1/CIP1) stability by WISp39, a Hsp90 binding TPR protein. Mol Cell 2005; 17: 237-249.

7. Seoane J, Le HV, Massague J. Myc suppression of the p21(Cip1) Cdk inhibitor influences the outcome of the p53 response to DNA damage. Nature 2002; 419: 729-734.

8. Wouters BG, Giaccia AJ, Denko NC, Brown JM. Loss of p21Waf1/Cip1 sensitizes tumors to radiation by an apoptosis-independent mechanism. Cancer Res 1997; 57: 4703-4706. 
9. Weiss RH. p21Waf1/Cip1 as a therapeutic target in breast and other cancers. Cancer Cell 2003; 4: 425-429

10. Kawabe T. G2 checkpoint abrogators as anticancer drugs. Mol Cancer Ther 2004; 3: 513-519.

11. Graves PR, Yu L, Schwarz JK, Gales J, Sausville EA, O'Connor PM et al. The Chk1 protein kinase and the $\mathrm{Cdc} 25 \mathrm{C}$ regulatory pathways are targets of the anticancer agent $\mathrm{UCN}-01$. J Biol Chem 2000; 275: 5600-5605.

12. Tse AN, Schwartz GK. Potentiation of cytotoxicity of topoisomerase i poison by concurrent and sequential treatment with the checkpoint inhibitor UCN-01 involves disparate mechanisms resulting in either p53-independent clonogenic suppression or p53dependent mitotic catastrophe. Cancer Res 2004; 64: 6635-6644.

13. Bunz F, Hwang PM, Torrance $C$, Waldman T, Zhang Y, Dillehay L et al. Disruption of p53 in human cancer cells alters the responses to therapeutic agents. J Clin Invest 1999; 104 263-269.

14. Beuvink I, Boulay A, Fumagalli S, Zilbermann F, Ruetz S, O'Reilly T et al. The mTOR inhibitor RAD001 sensitizes tumor cells to DNA-damaged induced apoptosis through inhibition of p21 translation. Cell 2005; 120: 747-759.

15. Simon JA, Lange CA. Roles of the EZH2 histone methyltransferase in cancer epigenetics. Mutat Res 2008; 647: 21-29.

16. Bracken AP, Helin K. Polycomb group proteins: navigators of lineage pathways led astray in cancer. Nat Rev Cancer 2009; 9: 773-784.

17. Varambally S, Dhanasekaran SM, Zhou M, Barrette TR, Kumar-Sinha C, Sanda MG et al. The polycomb group protein EZH2 is involved in progression of prostate cancer. Nature 2002; 419: 624-629

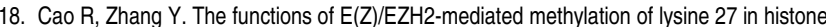
H3. Curr Opin Genet Dev 2004; 14: 155-164.

19. Cao Q, Yu J, Dhanasekaran SM, Kim JH, Mani RS, Tomlins SA et al. Repression of E-cadherin by the polycomb group protein EZH2 in cancer. Oncogene 2008; 27: 7274-7284.

20. Fujii S, Ito K, Ito $\mathrm{Y}$, Ochiai A. Enhancer of zeste homologue 2 (EZH2) downregulates RUNX3 by increasing histone $\mathrm{H} 3$ methylation. J Biol Chem 2008; 283 : 17324-17332.

21. Yang X, Karuturi RK, Sun F, Aau M, Yu K, Shao R et al. CDKN1C (p57) is a direct target of EZH2 and suppressed by multiple epigenetic mechanisms in breast cancer cells. PLoS One 2009; 4: e5011.

22. Bracken AP, Pasini D, Capra M, Prosperini E, Colli E, Helin K. EZH2 is downstream of the pRB-E2F pathway, essential for proliferation and amplified in cancer. Embo J 2003; 22 5323-5335

23. Chen $\mathrm{H}, \mathrm{Tu} \mathrm{SW}$, Hsieh JT. Down-regulation of human DAB2IP gene expression mediated by polycomb Ezh2 complex and histone deacetylase in prostate cancer. J Biol Chem 2005; 280: 22437-22444.

24. Min J, Zaslavsky A, Fedele G, McLaughlin SK, Reczek EE, De Raedt T et al. An oncogenetumor suppressor cascade drives metastatic prostate cancer by coordinately activating Ras and nuclear factor-kappaB. Nat Med 2010; 16: 286-294.

25. Kleer CG, Cao Q, Varambally S, Shen R, Ota I, Tomlins SA et al. EZH2 is a marker of aggressive breast cancer and promotes neoplastic transformation of breast epithelial cells. Proc Natl Acad Sci USA 2003; 100: 11606-11611.

26. Bracken AP, Kleine-Kohlbrecher D, Dietrich N, Pasini D, Gargiulo G, Beekman C et al. The Polycomb group proteins bind throughout the INK4A-ARF locus and are disassociated in senescent cells. Genes Dev 2007; 21: 525-530.

27. Yu J, Cao Q, Mehra R, Laxman B, Yu J, Tomlins SA et al. Integrative genomics analysis reveals silencing of beta-adrenergic signaling by polycomb in prostate cancer. Cancer $\mathrm{Cell}$ 2007; 12: 419-431.
28. Wu ZL, Zheng SS, Li ZM, Qiao YY, Aau MY, Yu Q. Polycomb protein EZH2 regulates E2F1-dependent apoptosis through epigenetically modulating Bim expression. Cell Death Differ 2009; 17: 801-810.

29. Rodriguez R, Meuth M. Chk1 and p21 cooperate to prevent apoptosis during DNA replication fork stress. Mol Biol Cell 2006; 17: 402-412.

30. Abbas T, Dutta A. p21 in cancer: intricate networks and multiple activities. Nat Rev Cancer 2009; 9: 400-414.

31. Ho MS, Tsai PI, Chien CT. F-box proteins: the key to protein degradation. J Biomed Sci 2006; 13: 181-191.

32. Hermand D. F-box proteins: more than baits for the SCF? Cell Div 2006; 1: 30

33. Tan J, Yang X, Zhuang L, Jiang X, Chen W, Lee PL et al. Pharmacologic disruption of Polycomb-repressive complex 2-mediated gene repression selectively induces apoptosis in cancer cells. Genes Dev 2007; 21: 1050-1063.

34. Jariel-Encontre I, Bossis G. Piechaczyk M. Ubiquitin-independent degradation of proteins by the proteasome. Biochim Biophys Acta 2008; 1786: 153-177.

35. Chen X, Barton LF, Chi Y, Clurman BE, Roberts JM. Ubiquitin-independent degradation of cell-cycle inhibitors by the REGgamma proteasome. Mol Cell 2007; 26: 843-852.

36. Waldman T, Lengauer C, Kinzler KW, Vogelstein B. Uncoupling of $S$ phase and mitosis induced by anticancer agents in cells lacking p21. Nature 1996; 381: 713-716.

37. Bunz F, Fauth C, Speicher MR, Dutriaux A, Sedivy JM, Kinzler KW et al. Targeted inactivation of p53 in human cells does not result in aneuploidy. Cancer Res 2002; 62: 1129-1133.

38. Lapenna S, Giordano A. Cell cycle kinases as therapeutic targets for cancer. Nat Rev Drug Discov 2009; 8: 547-566.

39. Bucher N, Britten CD. G2 checkpoint abrogation and checkpoint kinase-1 targeting in the treatment of cancer. Br J Cancer 2008; 98: 523-528.

40. Koniaras K, Cuddihy AR, Christopoulos H, Hogg A, O'Connell MJ. Inhibition of Chk1dependent G2 DNA damage checkpoint radiosensitizes p53 mutant human cells. Oncogene 2001; 20: 7453-7463.

41. Zhou BB, Bartek J. Targeting the checkpoint kinases: chemosensitization versus chemoprotection. Nat Rev Cancer 2004; 4: 216-225.

42. Ashwell S, Zabludoff S. DNA damage detection and repair pathways-recent advances with inhibitors of checkpoint kinases in cancer therapy. Clin Cancer Res 2008; 14: 4032-4037.

43. Suva ML, Riggi N, Janiszewska M, Radovanovic I, Provero P, Stehle JC et al. EZH2 is essential for glioblastoma cancer stem cell maintenance. Cancer Res 2009; 69: 9211-9218.

44. Iliopoulos D, Lindahl-Allen M, Polytarchou C, Hirsch HA, Tsichlis PN, Struhl K. Loss of miR-200 inhibition of Suz12 leads to polycomb-mediated repression required for the formation and maintenance of cancer stem cells. Mol Cell 2010; 39: 761-772.

45. Viale A, De Franco F, Orleth A, Cambiaghi V, Giuliani V, Bossi D et al. Cell-cycle restriction limits DNA damage and maintains self-renewal of leukaemia stem cells. Nature 2009; 457: $51-56$.

46. Dean M, Fojo T, Bates S. Tumour stem cells and drug resistance. Nat Rev Cancer 2005; 5: 275-284.

47. Kadota M, Yang HH, Gomez B, Sato M, Clifford RJ, Meerzaman D et al. Delineating genetic alterations for tumor progression in the MCF10A series of breast cancer cell lines. PLoS One 2010; 5: e9201.

48. Sheaff RJ, Singer JD, Swanger J, Smitherman M, Roberts JM, Clurman BE. Proteasoma turnover of p21Cip1 does not require p21Cip1 ubiquitination. Mol Cell 2000; 5: 403-410.

49. Jiang X, Tan J, Li J, Kivimae S, Yang X, Zhuang L et al. DACT3 is an epigenetic regulator of Wnt/beta-catenin signaling in colorectal cancer and is a therapeutic target of histone modifications. Cancer Cell 2008; 13: 529-541.

50. Santra MK, Wajapeyee N, Green MR. F-box protein FBXO31 mediates cyclin D1 degradation to induce G1 arrest after DNA damage. Nature 2009; 459: 722-725.

Supplementary Information accompanies the paper on Cell Death and Differentiation website (http://www.nature.com/cdd) 